\title{
A stochastic model for the aerodynamics of irregularly shaped gravel
}

\author{
Md Safwan Ahsanullah ${ }^{\mathrm{a},}{ }^{*}$, Nigel B Kaye ${ }^{\mathrm{b}}$ \\ ${ }^{a}$ Glenn Department of Civil Engineering, Clemson University, Clemson, SC, USA, \\ mahsanu@clemson.edu \\ ${ }^{b}$ Glenn Department of Civil Engineering, Clemson University, Clemson, SC, USA, \\ nbkaye@clemson.edu
}

\begin{abstract}
:
Damage from hurricanes hitting the U.S. between 1980 and 2018 totaled $\$ 862$ billion (NHC). Post-storm investigations show wind-borne debris is a major contributor to total economic loss. This paper investigates the flight of compact wind-borne debris which has previously only been treated as spherical, ignoring lift forces and shape irregularity, resulting in a two-dimensional flight. However, the trajectory of a piece of gravel is not two-dimensional as its orientation changes during flight, altering the drag force and generating lift forces. This study proposes a stochastic model to resolve some key aspects of the trajectory due to the change in orientation of the debris particle during its flight. The model shows good agreement with experiments. Improved modeling of compact debris will improve our understanding of the risk of damage from windborne debris and enable improved mitigation measures resulting in more resilient communities.
\end{abstract}

Keywords: compact debris, debris flight, numerical, stochastic model, gravel, windborne debris

\section{INTRODUCTION}

Wind-borne debris and missiles in events of severe windstorms, hurricanes and other strong wind events have been observed to cause significant damage to the built environment. Reports after notable wind events show that the wind-borne debris had been a major contributor to the total economic loss (Minor, 2005). To address the issue of damage from wind-borne debris, building design codes have gone through several modifications over time. However, there still exists a significant knowledge gap around the motion initiation and resulting flight of a gravel. These problems can be solved only with a deeper understanding of the forces acting on particles of random shapes.

The standard flight equations treat debris particles as spheres (Baker, 2007; Holmes, 2004) and fail to model the stochastic nature of actual debris flight. This study proposes a stochastic model in an attempt to resolve some key aspects of the stochastic nature of the flight that originates due to the change in orientation of the debris particle during its flight, and, as a result, the alteration of the projected cross-sectional area, the lift and the drag coefficients.

\section{EXPERIMENTS AND OBSERVATIONS}

To gain insight on the motion of irregularly shaped gravel pieces moving through a fluid, a simple experimental setup is designed for this study. The setup consists of a clear-sided tank filled with water and gravel pieces of different sizes as representative of a typical compact debris. The main objective of the experiment is to observe the spread and the radial distances of the landing locations 
of dropped gravel pieces. Five gravel sizes are used in the drop experiments while a total of 200 gravel pieces were dropped per gravel size.

The gravel pieces are observed to change their orientation during their flight, leading to alteration of drag forces and generation of lift forces, and their trajectories are neither linear, vertical nor self-repeating. All these findings contradict the underlying assumptions of standard debris flight equations. Due to these factors, the landing locations of the gravel pieces spread around the center, with a finite mean and standard deviation of radial distances of landing locations from the center of the base. This is qualitatively similar to the drop experiments for rod-like debris (Tohidi and Kaye, 2017).

\section{MODEL DEVELOPMENT}

The proposed stochastic model numerically solves the following coupled three-dimensional rectilinear differential equations that govern the motion of debris particles:

$$
\begin{aligned}
& \frac{d^{2} \mathbf{x}}{d t^{2}}=\frac{\sum \mathbf{F}}{m} \\
& \frac{d \mathbf{x}}{d t}=\mathbf{u}
\end{aligned}
$$

where, $\mathbf{x}$ is the position vector, $\mathbf{u}$ is the velocity vector, $\Sigma \mathbf{F}$ is the net force acting on a debris particle and $m$ is the mass of a debris particle. The bolded symbols here represent Cartesian vectors. The net force in Eq. 1 is the resultant of constant weight and buoyancy forces and varying drag and lift forces. The model randomly varies the drag and lift forces at each time-step during the debris flight by perturbing the non-dimensional projected area of the gravel piece $(\alpha)$, the aerodynamic force coefficients $\left(\mathrm{C}_{\mathrm{D}}\right.$ and $\left.\mathrm{C}_{\mathrm{L}}\right)$ and the lift force direction, $\mathbf{n}_{\mathbf{L}}$. The overall range of $\mathrm{C}_{\mathrm{D}}, \mathrm{C}_{\mathrm{L}}$ and $\alpha$ are determined based on wind tunnel measurements of the force coefficients (Chai et al, 2019) and laboratory measurements of gravel geometry. The lift force direction is altered by randomly perturbing the direction of the reference lift angle $(\theta)$ within the range $0^{\circ} \leq \theta \leq 60^{\circ}$.

\section{MODEL RESULTS AND COMPARISON}

The model varies the magnitudes of perturbations $\left(\delta \mathrm{C}_{\mathrm{D}}, \delta \mathrm{C}_{\mathrm{L}}, \delta \theta\right.$ and $\left.\delta \alpha\right)$ of each of the varying parameters within their global ranges and simulates gravel drops for different combinations of perturbation magnitudes. The perturbation ranges $\delta C_{D}, \delta C_{L}$ and $\delta \alpha$ were varied from 0 to $50 \%$ of the overall range of the respective parameter in increments of $2.5 \%$ of the overall range while $\delta \theta$ was varied from $0^{\circ}$ to $60^{\circ}$ in $1.5^{\circ}$ increments. This leads to a total of $41 \times 21^{3}$ possible combinations, and for each combination, simulation of 200 drops per gravel size leads to approximately 380 million drop simulations. Finally, an optimized combination of perturbation parameters, $\left(\delta \mathrm{C}_{\mathrm{D}}, \delta \mathrm{C}_{\mathrm{L}}, \delta \theta, \delta \alpha\right)_{\text {opt }}$, is obtained that results in good agreement between the numerical and experimental range of radial distances of landing locations. Fig. 1 shows how the numerical spread of landing locations for gradation A (largest gradation) and ranked radial distances compare to those obtained from the experiments. The solid line in the right-hand side figure shows perfect agreement line of unit slope. 

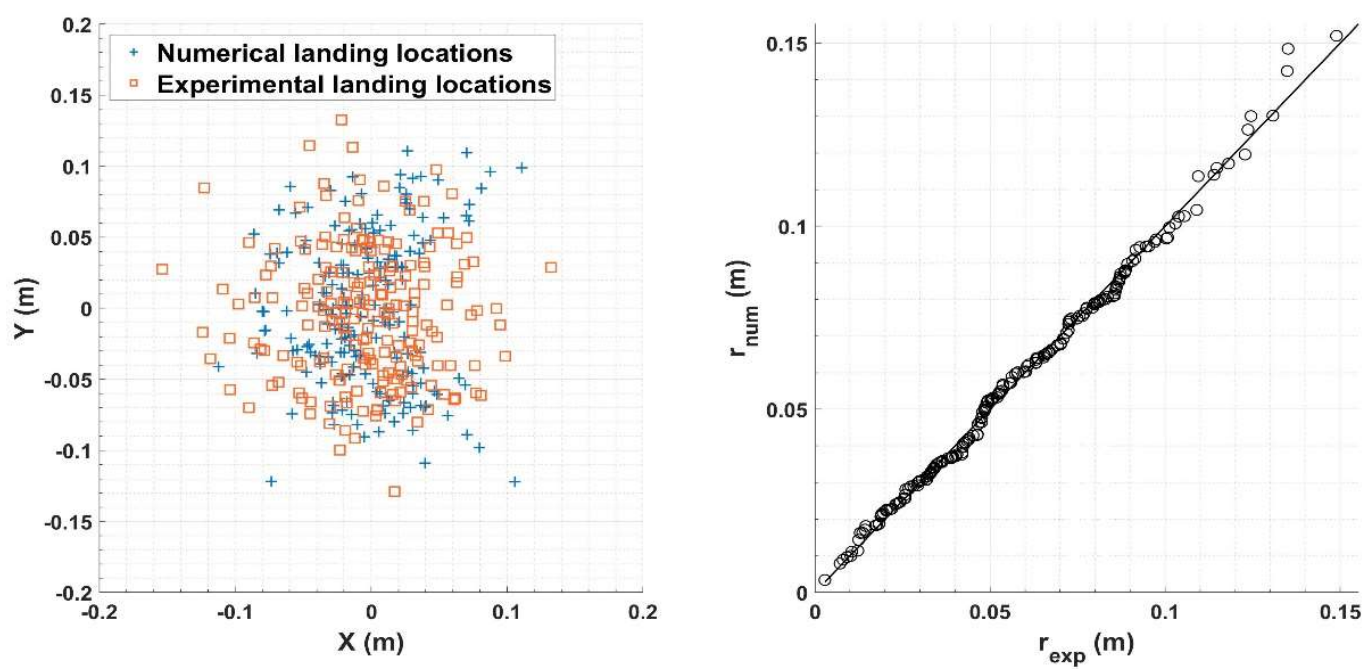

Figure 1. Comparison between numerical and experimental spreads of landing locations and ranked radial distances from the model against those from experiments. (Gradation A)

Gaining a thorough understanding of the motion of windborne debris can bring great benefit for us to reduce the impact of such debris during extreme wind events. From accurate predictions of landing locations of flying debris and missiles, we can take preemptive measures to reduce the overall loss of property and lives in case of such extreme events.

\section{ACKNOWLEDGEMENTS}

The authors gratefully acknowledge the insights provided by Dr. Abdul A. Khan and Dr. William C. Bridges Jr on different aspects of this study during a discussion session. They also thank Megan Holmes for her work on the experiments. This material is based upon work supported by the National Science Foundation under Grant No. 1760999. Any opinions, findings, and conclusions or recommendations expressed in the material are those of the author and do not necessarily reflect the views of the NSF.

\section{REFERENCES}

Baker, C. J., 2007. The debris flight equations. Journal of Wind Engineering and Industrial Aerodynamics, $329-353$.

Chai, V., Parkhi, D., Boopathy, S., Xiang, J. and Schlüter, J., 2019. A model for the aerodynamic coefficients of rocklike debris. Comptes Rendus Mecanique, 19-32.

Holmes, J., 2004. Trajectories of spheres in strong winds with application to wind-borne debris. Journal of Wind Engineering and Industrial Aerodynamics, 9-22.

Minor, J. E., 2005. Lessons learned from failures of the building envelope. Journal of Architectural Engineering, 1013.

Tohidi, A. and Kaye, N. B., 2017. Aerodynamic characterization of rod-like debris with application to firebrand transport. Journal of Wind Engineering and Industrial Aerodynamics, 297-311. 